\title{
Study of the Smoke Propagation in a Road Tunnel
}

\author{
B. Kalech*, B. Jouini, M. Bouterra, A. el Cafsi, and A. Belghith
}

LETTM, Faculty of Sciences of Tunis, El Manar 2092, University of Tunis-El Manar, Tunisia

Received 20 April 2013, accepted in final revised form 22 December 2013

\begin{abstract}
Road tunnels are increasingly built due to heavy traffic in dense urban areas. Knowledge of the spread of smoke is still limited. A numerical study on the smoke flow in a tunnel has been made using the model of small-scale tunnel. Effects of the longitudinal air flow on the flow structure, the temperature distribution and the smoke stratification of the fire in the tunnel have been considered by taking into account the inclination of the tunnel and the transverse positioning of the source. The results show that the spread of smoke is independent of the transverse position of the source in the fourth phase of the flow. The slope has a slight influence on the flow and promotes the flow stratification in a ascending slope. The longitudinal air flow promotes destratification of the smoke flow. The model was validated by comparison with experimental data obtained from fire tests (Wu et al.) in a smallscale tunnel.
\end{abstract}

Keywords: Tunnel fire; Smoke flow; Stratification; FDS

(C) 2014 JSR Publications. ISSN: 2070-0237 (Print); 2070-0245 (Online). All rights reserved.

doi: http://dx.doi.org/10.3329/jsr.v6i1.14696 J. Sci. Res. 6 (1), 51-68 (2014)

\section{Introduction}

The understanding and description of the physical phenomena of smoke propagation in tunnels is necessary for the assessment of risks of a fire. This study presents the numerical approach of the propagation of smoke in a typical configuration on a small-scale. The geometrical configuration of study is at ratio of 1: 20 with respect to real scale. The issues raised by the propagation phenomenon based on the fact that smoke is a vector of transporting energy and matter can cause disastrous fires in road tunnels. The smoke is a dynamic entity since it is constantly in motion, it changes of composition and temperature at any time and it moves conditions of the environment. Like all fluids, smoke flows under the action of external forces, which are essentially the buoyancy and gravity.

Overpressure caused by fire, thermal expansion, the presence of winds, openings, systems airflow, density and composition of the smoke are parameters that affect the flow

\footnotetext{
*Corresponding author: ib.kalech.fst@gmail.com
} 
of fumes. For all these reasons, it is very difficult to predict how the smoke flows in a fire, and the characteristics of a tunnel fire vary from one case to another.

When a fire broke out in a tunnel, the development of the flow of a layer of smoke floating in a tunnel can be summarized in four phases [1]:

- Impinging region of rising plume on the ceiling

- Radial spread of smoke under the ceiling after impingement

- Interaction with side walls, and thus the transition region to one-dimensional spread

- One-dimensional spreading.

A theoretical model of a tunnel fire is proposed by JP Kunsch [2] to describe the behavior of smoke in the opposite direction to a current of fresh air induced by the tunnel ventilation. It is shown that the critical ventilation velocity depends mainly on the power of the fire and the height of the tunnel. Also adding to the large fires, the critical speed does not depend on the power.

Brahim et al. [3] proposed numerical investigations carried out on a small scale tunnel model to study the fire-induced smoke control by longitudinal and longitudinal-natural ventilation systems. They are studied the effect of two ventilation systems on the temperature distribution and the flow stratification to estimate the effectiveness of ventilation systems. The longitudinal velocity affects the thermal stratification especially: firstly, longitudinal velocity generally results in the decrease of the hot layer temperature and increase of the longitudinal velocity of upper buoyant flow, by enhancing the heat and mass exchange between the hot stratified layer and the lower cool layer. Secondly, longitudinal velocity air flow increases the mixture between the upper hot layer and the lower cool layer.

Experimental studies on smoke in a tunnel sloping are performed by the model of tunnel scale [4]. The results show that the spread of smoke up much faster with increasing slope gradient, and a thick layer of smoke was formed during movement of smoke in the bottom of the tunnel and fills. The velocity of ventilation had a great influence on the temperature distribution along the tunnel and the smoke stratification will be distributed when the speed of longitudinal ventilation.

Tajadura et al. [5] examined the effect of the slope on the extraction of the pollutant in a long road tunnel $(1535 \mathrm{~m})$ existing in Barcelona. For more efficient extraction, it was determined that the exhaust sections must be opened up to the location of the fire. Atkinson et al. [6] presented experimental results from a study involving the design of tunnels with slopes between 0 and 10 degrees to deduce the critical velocity and examine the effect of the slope on the latter. For tunnels with a glide slope, the critical velocity is slightly greater than for the corresponding horizontal tunnel.

Lee and Ryou [7] performed numerical simulations to validate the FDS code to examine the effect of the cross-sectional area of the tunnel on the movement of smoke from fires in tunnels with longitudinal ventilation system. The study was conducted on a smallscale with different ignition sources ranging from $2 \mathrm{KW}$ to $12 \mathrm{KW}$. The extension of the tunnel section affects the growth and development of smoke in tunnel fires. They confirmed by the analysis of temperature fields and velocity, the smoke moves at high 
temperatures along the ceiling and fresh air flows along the bottom of the tunnel. Temperature near the fire source decreased with increasing the aspect ratio $(\mathrm{H} / \mathrm{W})$.

$\mathrm{Hu}$ et al. [8] performed fire tests at full scale in a long channel of $88 \mathrm{~m}$, to study variation in the temperature and the speed of the smoke. The results show that the temperature distribution along the channel decreases exponentially with a factor of about 0.035 . The velocity distribution along the channel is shown quite well by exponential equations.

A series of experiments were carried by $\mathrm{Ji}$ et al. [9] to investigate the influence of different places cross fire on the maximum temperature in the tunnel ceiling. The results show that the restrictive effect of the side walls of the tunnel causes the temperature increase to the maximum compared with the open space. The maximum temperature of smoke above the fire is almost unchanged and then increases significantly if the distance between the fire and the side wall decreases to a certain value.

A set of experiments with fire n-heptane were conducted to study the mode of air entrainment in road tunnels with natural ventilation [10]. The results show that the use of vertical wells to remove fumes leads to a strong mixing process between the smoke layer and the fresh air. In addition, a portion of entrained air mixes into the smoke layer below, which also proves ineffective smoke.

Blanchard et al. [11] have experimentally and numerically studied a tunnel reduced scale (1/3), using a pool fire heptane until $4 \mathrm{MW}$. Efforts were made to verify the validity of the simulation compared with the experimental databases. For example, an analysis showed that the energy balance is heavily dependent on the ventilation system. In fact, approximately $50 \%$ and $67 \%$ of the heat is dissipated through interaction with the wall in the sub-critical and critical regimes.

Two sets of model scale experimental tests were also carried out by Li et al. [12]. The heat, longitudinal velocity and the parameters of geometry tunnel are taken into account. The results of analysis and experiments show that the maximum temperature excess gas in the ceiling and the temperature can be divided into two regions. When the dimensionless ventilation velocity is greater than 0.19 , the maximum temperature is below the ceiling of the tunnel, it increases linearly with the heat release rate and decreases linearly with the speed of longitudinal ventilation. When the dimensionless velocity ventilation is less than 0.19 , the maximum temperature under the ceiling of the gas varies as the third power of two velocity dimensionless heat release, independently of the longitudinal velocity of ventilation.

Yang et al. [13] have performed experiments in a horizontal channel to a reduced scale to investigate the behavior of the flow stratification caused by the fire, as a result of the mechanical force characterized by the rate of aspiration opening in the ceiling of channel. The horizontal velocity of the movement, the vertical profile of the temperature and the height of the interface of stratification of the flow dynamics were measured. Based on the Froude number and Richardson, they proved that the model is divided into three stratification regions. 
Chen [14] made an analytical study aimed to understand the smoke propagation of fire in road tunnels. This review article includes 60 references. The analytical results indicate that, in the upstream of fire, the critical ventilation velocity is generally proportional to the one-third power of the heat release rate (HRR); some modifications to this power law are necessary for special cases. In the downstream of fire, the smoke propagates along the tunnel with a constant speed, which is essentially linearly proportional to the ventilation velocity. The numerical results determine a safety domain in terms of the ventilation velocity and the HRR of fire.

The general case of a fire is always accompanied by the presence of a velocity component transverse to the smoke flow. Taking account of the longitudinal air flow, we study the effects of transverse positioning of the source and the slope on the structure of the flow, the temperature distribution and stratification of fire smoke in the tunnel. And among undeclared goals is taken into account these important factors in the design of a ventilation strategy.

\section{Numerical Investigation}

To characterize the behavior of the spread of smoke, the numerical tests of fire were performed. The influence of the longitudinal velocity of the smoke behavior between different fire sizes (powers) was considered.

\subsection{Numerical model}

In recent years, the application code "FDS" has been generalized as fire simulation tool. This technique has reached the tunnel applications where the calculations are now part of many researches, evaluations and investigations. This results in a large number of recent publications in journals; he has also become a practice more common paratice in research. The Code FDS (Fire Dynamics Simulator) was developed by NIST to study fire behavior and evaluate the performance of systems for protection against fires in confined and semi confined environments. The computer program can be used to analyze problems related to fire, such as the distribution of temperature, velocity and concentration, FDS (version 5) code has been validated $[15,16]$ to be able to simulate the propagation of smoke and species transport in a confined environment.

Simulations require solving the full set of partial differential equations, conservation of mass, momentum and energy. The conservation equations for mass, momentum and energy for a Newtonian fluid are as follows [15].

Conservation of mass: $\frac{\partial \rho}{\partial t}+\nabla \cdot(\rho u)=m_{b}^{\prime \prime \prime}$

Conservation of momentum: $\left(\frac{\partial}{\partial t} \rho u\right)+\nabla \cdot \rho u u+\nabla p=\rho g+f_{b}+\nabla \cdot \tau$

Transport of Sensible Enthalpy: $\frac{\partial\left(\rho h_{S}\right)}{\partial t}+\nabla \cdot \rho h_{s} u-\frac{D p}{D t}=q^{\prime \prime \prime}+q^{\prime \prime \prime}{ }_{b}-\nabla \cdot q^{\prime \prime \prime}+\varepsilon$

Equation of State for a Perfect Gas: $p=\frac{\rho R T}{\bar{W}}$ 
The application of LES techniques to fire is aimed at extracting greater temporal and spatial fidelity from simulation of fire. This model explicitly calculates the turbulent large scales and models the effects of smaller ones using sub grid closure rules. The approach based on LES has a particular advantage over the Reynolds-averaging procedures in that only the effects of small-scale turbulence motion have to be modeled. The FDS code adopts the "low Mach number" combustion equations that describe the low speed motion of a gas driven by chemical heat release and buoyancy forces.

In predicting smoke movement by LES, two points should be considered $[15,16]$ :

- Fine enough grids; and

- A suitable Sub-grid model (SGM) on small eddies.

The LES Sub-grid model commonly used in LES was developed originally by Smagorinsky. A refined filtered dynamics sub-grid model was applied in the FDS model to account for the sub-grid scale motion of viscosity, thermal conductivity and material diffusivity [15]. The dynamic viscosity defined in FDS is:

$$
\mu_{L E S}=\rho(C S \Delta)^{2}\left(2 \overline{S_{l \jmath}} \overline{S_{l \jmath}}-\frac{2}{3}(\nabla \cdot \bar{u})^{2}\right)^{1 / 2}
$$

Where $C_{\mathrm{s}}$ is the empirical Smagorinsky constant, $\Delta=(\delta x \delta y \delta z)^{1 / 3}$ and

$$
\begin{aligned}
|S|=2\left(\frac{\partial u}{\partial x}\right)^{2}+ & 2\left(\frac{\partial v}{\partial y}\right)^{2}+2\left(\frac{\partial w}{\partial z}\right)^{2}+\left(\frac{\partial u}{\partial x}+\frac{\partial v}{\partial y}\right)^{2}+\left(\frac{\partial u}{\partial z}+\frac{\partial w}{\partial x}\right)^{2}+\left(\frac{\partial v}{\partial z}+\frac{\partial w}{\partial y}\right)^{2} \\
& -\frac{2}{3}(\nabla \cdot \vec{u})^{2}
\end{aligned}
$$

The term $|S|$ consists of second-order spatial differences averaged at the grid centre. The thermal conductivity $k_{\text {LES }}$ and material diffusivity $D_{\text {LES }}$ of the fluid are related to the viscosity $\mu_{\mathrm{LES}}$ in terms of the Prandtl number Pr and Schmidt number $S_{\mathrm{c}}$ by:

$$
k_{L E S}=\frac{C_{p} \mu_{L E S}}{P_{r}} ;(\rho D)_{l, L E S}=\frac{\mu_{L E S}}{S_{c}}
$$

Both $\operatorname{Pr}$ and $S_{\mathrm{c}}$ are assumed to be constant. The specific heat $\mathrm{Cp}$ is taken to be that of the dominant species of the mixture $[15,16]$.

The Courant-Friedrichs-Lewy (CFL) was used in FDS [15] for justifying convergence. This criterion is more important for large-scale calculations where convective transport dominates the diffusive one. The estimated velocities are tested at each time step to ensure that the CFL condition is satisfied [15]: $\max \left(\frac{\left|u_{i j k}\right|}{\delta x}, \frac{\left|v_{i j k}\right|}{\delta y}, \frac{\left|w_{i j k}\right|}{\delta z}\right)<1$

The time step is eventually changed to a quasi-steady value when the fire burns steadily. The results from a numerical analysis are sensitive to the grid size used. In this study, we are sure that the CFL condition is satisfied for the first time step in order to obtain a good convergence. 


\subsection{Numerical-experimental comparison}

The validation of the FDS code was undertaken initially by the developers of the program and also by other researchers and organizations $[15,16]$. To evaluate the potential of the modeling technique FDS, we take the experimental study by Wu and Bakar [17].

The experimental data are taken from tests in tunnels on a small scale in order to determine an empirical relationship between the power of the fire source and the longitudinal velocity of ventilation.

In each test performed by $\mathrm{Wu}$ and Bakar, a source with a constant of power was turned on in the tunnel. The longitudinal velocity was gradually increased until the length of the return flow, the phenomenon of back-layering, (measured from the heat source) is equal to $2.25 H$, where $H$ is the height of the tunnel. Wu and Bakar [17] recorded their critical velocity of ventilation with different standards, thanks to their use of the boundary condition "blow" (Fig. 1a). In the standard condition, the critical speed is recorded from the blow over the cross section of the tunnel. Wu and Bakar have made already placed a correction to develop.

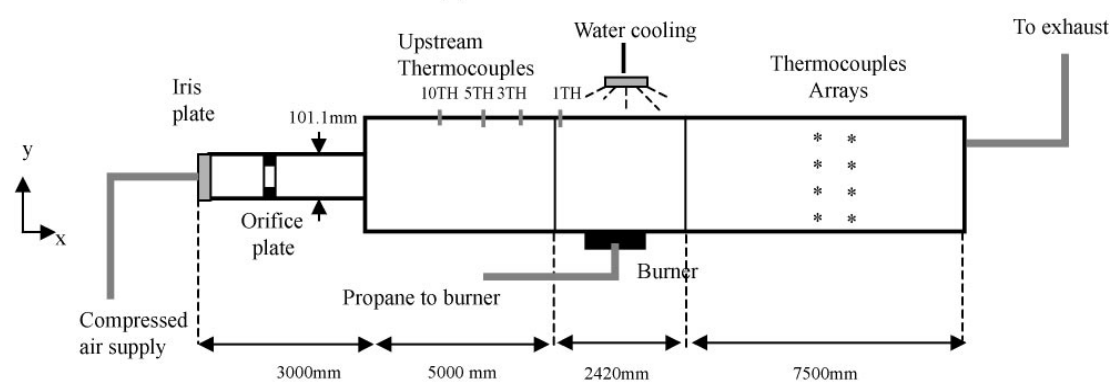

Fig. 1a. Experimental model of Wu and Bakar [16].

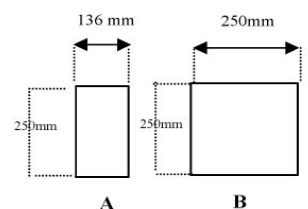

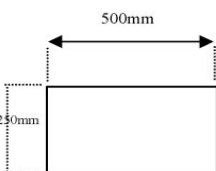

C

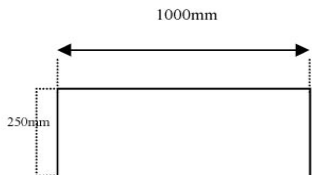

D

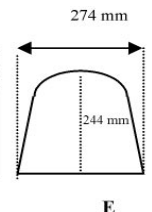

Fig. 1b. Sections of tunnels [16].

Tests were carried out on five different sections, Fig. 1b, and eight different power (1.5, $3,7.5,10.5,12,15,22.5$ and $30 \mathrm{~kW})$. The heat source is circular of diameter $106 \mathrm{~mm}$ $\left(0.0088 \mathrm{~m}^{2}\right)$. The fuel used is propane.

The hydraulic tunnel height is the characteristic length of study. The forms Dimensionless of power and the critical velocity of ventilation used are given by:

$$
Q^{\prime \prime}=\frac{Q}{\rho_{0} \cdot T_{0} \cdot c_{p} \cdot g^{1 / 2} \cdot \bar{H}^{5 / 2}} ; V_{c}^{\prime \prime}=\frac{U_{c}}{\sqrt{g \bar{H}}}
$$


Here $\rho_{0}$ is ambient air density, $C \mathrm{p}$ is heat capacity of the hot gases, $T_{0}$ is the ambient temperature and $\mathrm{g}$ the acceleration due to gravity $\left(\mathrm{g}=9.81 \mathrm{~m} / \mathrm{s}^{2}\right)$. The expression of $\bar{H}$ is $\bar{H}=4 . A / P$. A cross-sectional area of the tunnel and $\mathrm{P}$ is the tunnel perimeter.

The geometric configuration of the field study is shown in Fig. 1a. The longitudinal computational domain, $L=14.92 \mathrm{~m}$, was divided into three segments, Fig. 1b. Segment 1 is the upstream section of the tunnel, its length is $5.04 \mathrm{~m}$ and $\Delta x=0.03 \mathrm{~m}$. Segment 2 was the burner section of length $2.38 \mathrm{~m}$ and $\Delta x=0.01 \mathrm{~m}$. Segment 3 is the downstream section of the tunnel, its length is $7.50 \mathrm{~m}$ and $\Delta x=0.03 \mathrm{~m}$. Non-uniform distribution of the mesh allows us to maintain a sufficient degree of accuracy in the solution, was shown in figure 1c. Width, $W=0.5 \mathrm{~m}$, is meshed with $\Delta y=0.01 \mathrm{~m}$ and height, $\mathrm{H}=0.25 \mathrm{~m}$, is meshed with $\Delta z=0.01 \mathrm{~m}$.

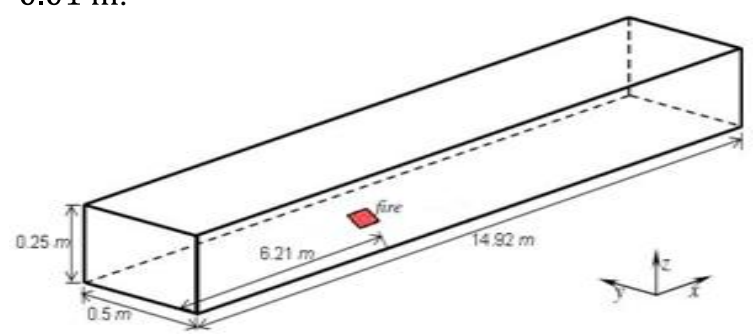

Fig. 2. Geometrical configuration (adopted for C).

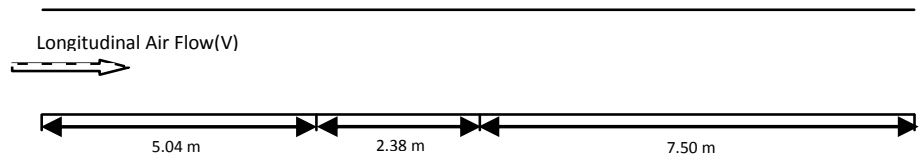

Fig. 3. Distribution of mesh.
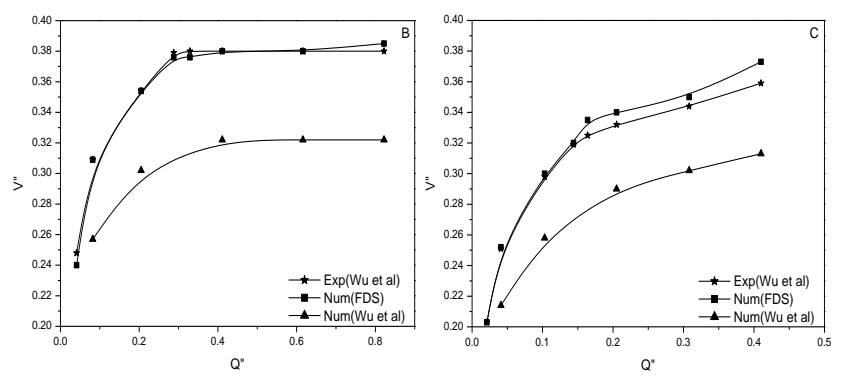

Fig. 4. The dimensionless critical velocities function of the dimensionless power, for two configurations (B and C).

The first longitudinal plane of the field was set at the entrance of the ventilation velocity and the last was set as opened naturally to the external environment. The circular burner was simulated as burner square shapes of different sizes suitable for every size of 
fire. The length of the source varies from 0.08 to $0.26 \mathrm{~m}$ for power going from 1.5 to 30 $\mathrm{KW}$. These sizes correspond to fires power from 2.5 to $50 \mathrm{MW}$ in reality. A propane gas burner was used to simulate the fire source, to designate a rate of heat release per unit area (HRRPUA) on the SURF line. Fuel properties "propane" is taken from the bibliography of FDS, the Prandtl number was adjusted from 0.5 to 0.2 . The square section (B) and rectangular $(\mathrm{C})$ have been fully taken into account.

The numerical and experimental results for the configuration and B are shown in Fig. 4. The recorded values of our numerical simulation for the critical speed of ventilation have an error rate as a percentage ranging from $1.5 \%(0.6-0.61 \mathrm{~m} / \mathrm{s})$ to $4 \%(0.39-0.38 \mathrm{~m} / \mathrm{s})$. There are also other values that were found by point $(Q=3,7.5,10.5,15,22.5 \mathrm{KW})$.

The numerical and experimental results for the configuration $\mathrm{C}$ are given in Fig. 4. The recorded values of our numerical simulation for the critical speed of ventilation have an error rate percentage will $2 \%(0.45-0.47 \mathrm{~m} / \mathrm{s})$ to $4 \%(0.65-0.67 \mathrm{~m} / \mathrm{s})$. Also other values were found by point.

The numerical results of validation of $\mathrm{Wu}$ and al. are a percentage error of $18 \%$. Our numerical results are much more accurate than those of Wu et al. [17]. The error does not exceed 5\% that expresses the good agreement with experiment. The profiles found in our study are in good agreement with the experimental results. These results show the potential of the FDS code, specialized in the field of fire from the Fluent code.

\subsection{Configuration for simulation}

In comparing the results, we adjusted the parameters in the calculation FDS code, the constants of the turbulence and especially the mesh. The arrangement adopted is similar to that proposed by $\mathrm{Wu}$ and al to evaluate the characteristics of the flow under the influence of the slope and position of the source with taking into account the longitudinal air flow. The dimensions of the geometrical configuration have been modified. Fig. 6 shows the device adopted in this work. The source is in the center of the channel, it is of square shape with a length of $10 \mathrm{~cm}$. Fig. 7 shows the distribution of the mesh to study the problem.

Figs. 8 and 9 show the study parameters, respectively the application of longitudinal air flow, the slope (from $-5^{\circ}$ to $5^{\circ}$ ) and the transverse position of the source $(y=0.25, \mathrm{i} ; 0.20$, $\mathrm{j}$ and $0.10, \mathrm{k}) \mathrm{cm}$.

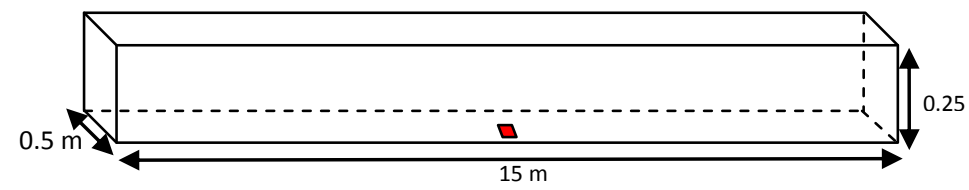

Fig. 6. Geometrical configuration adopted for simulation.

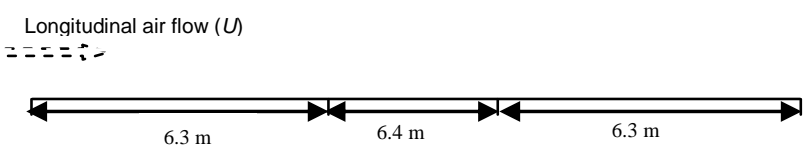

Fig. 7. Distribution of the mesh. 


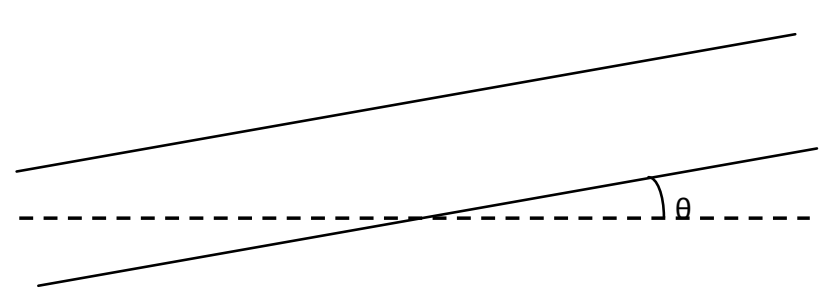

Fig. 8. Variation of the slope.

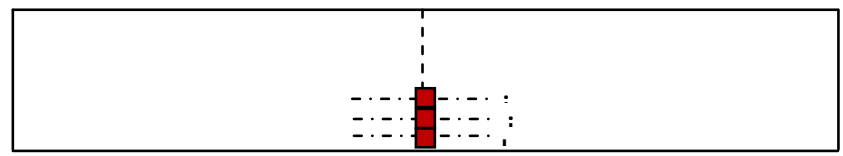

Fig. 9. Variation of transversal position of the source.

We propose to define a suitable method to quantify the characteristics of the flow generated in a fire tunnel ground (general case), in a rectangular channel. The study is focused on the unidirectional flow. Initially, we studied the stability and level of stratification of a layer of smoke generated from the ceiling without longitudinal velocity of fresh air. The study focuses on the variation of the slope and to its importance and the lack of knowledge about the spread of smoke in a sloping tunnel.

In previous studies, the fires were still supposed to happen to the longitudinal axis of the tunnel. The fires occur at all locations in tunnels with different distances from the wall, this study focuses on the transversal position of the source. In a second step, a longitudinal velocity variable is applied. The analysis of the stratification is manifested by the temperature distribution and analysis of the Richardson number.

\section{Results and Discussion}

The majority of tunnels have a slope more or less important, depending on their geographical location. And generally all urban tunnels are slightly inclined.
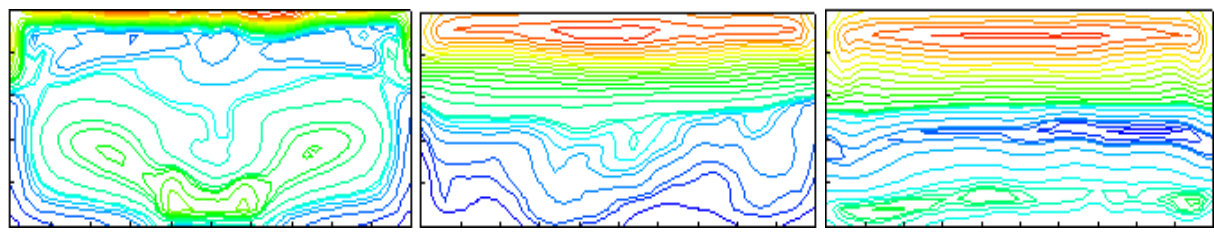

a) slope $=2.5^{\circ}$ 

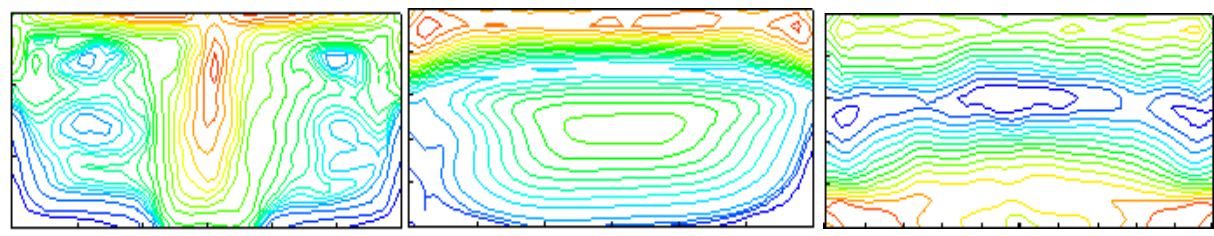

b) slope $=0^{\circ}$

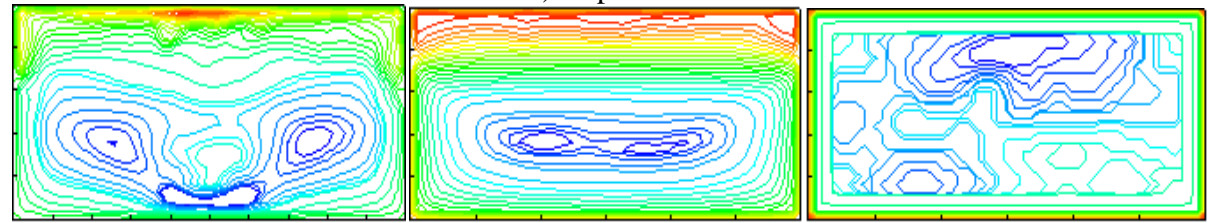

c) slope $=-2.5^{\circ}$

Fig. 10. Vortex fields with different slope, $x=7.5,9$ and $14.5 \mathrm{~m}$.

Sometimes, the slope is imposed in order to control the flow movement of fire smoke in tunnels. Fig. 10 shows that the smoke spreads much more quickly to the exit from the top due to the effect of the slope in the first level, and a thick layer of smoke is displaced in the top of the tunnel and filled it. The smoke rises by gravity and is therefore inclined to follow the slope of the tunnel to lead naturally to points of extraction (extraction by openings or mechanical ends of the tunnel).

Fig. 11 shows that there is a higher smoke layer and another layer below in all cases. In the absence of longitudinal velocity (Fig. 11a), there are two areas of recirculation on both sides of the plume. At $x=9 \mathrm{~m}$, the flow is divided into three zones, the layer of smoke and the intermediate layer and the layer of fresh air. The intermediate layer is thin thanks to the stability of the flow. At the downstream channel, $x=14.5 \mathrm{~m}$, we find the presence of three zones as the first layer of smoke, the second is the air entrainment (the shear) and the third is that of the flow of fresh air.

By applying a longitudinal air flow, $U=0.23 \mathrm{~m} / \mathrm{s}$, we observe the presence of two vortices that are related to the plume. The size of the vortices decreases on the ceiling. At $x$ $=9 \mathrm{~m}$, the flow is disturbed, the fresh air is driven by the lateral sides. At the downstream channel, the three zones are clearly visible. By increasing the intensity of the longitudinal air flow, $U=0.46 \mathrm{~m} / \mathrm{s}$, there are two separate recirculation zones and the flow is disturbed always below the ceiling. Then the disturbance affects all sections of the canal. Downstream, we find that the flow is homogeneous.
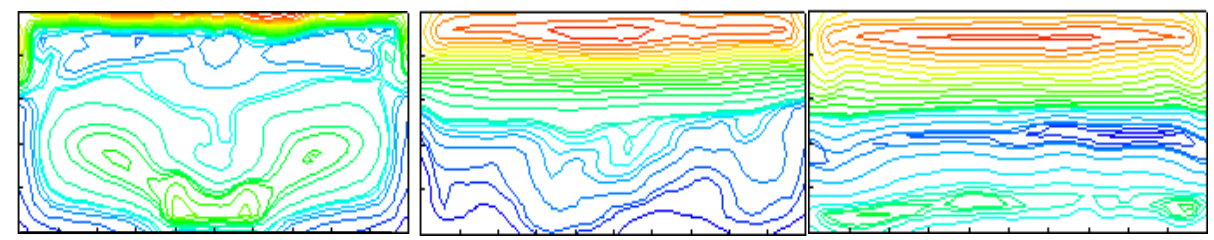

a) $U=0 \mathrm{~m} / \mathrm{s}$ 

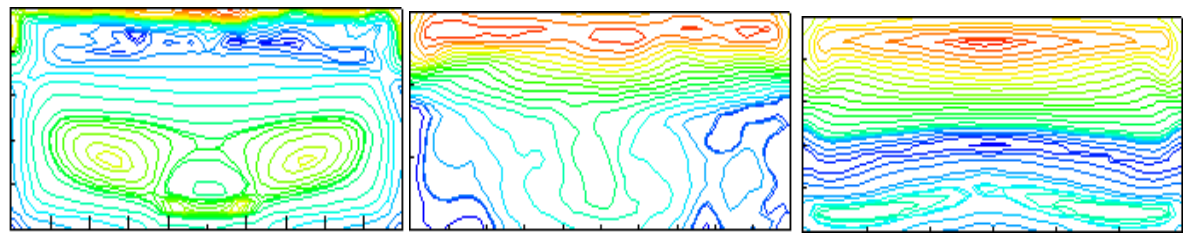

b) $U=0.23 \mathrm{~m} / \mathrm{s}$
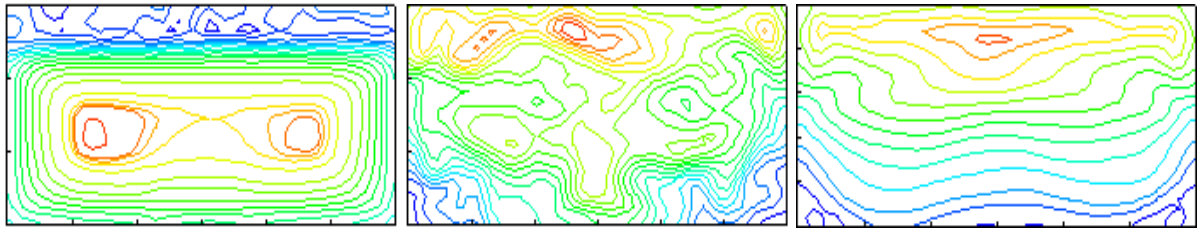

c) $U=0.46 \mathrm{~m} / \mathrm{s}$

Fig.11. Vortex fields with different longitudinal velocity for a slope equal to $2.5^{\circ}(\mathrm{x}=7.5,9$ and 14.5 $\mathrm{m})$.
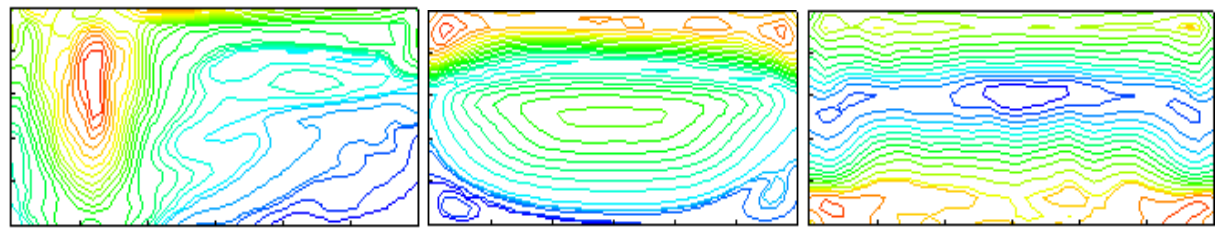

a) $\mathrm{S} 2(\mathrm{k}), U=0 \mathrm{~m} / \mathrm{s}$
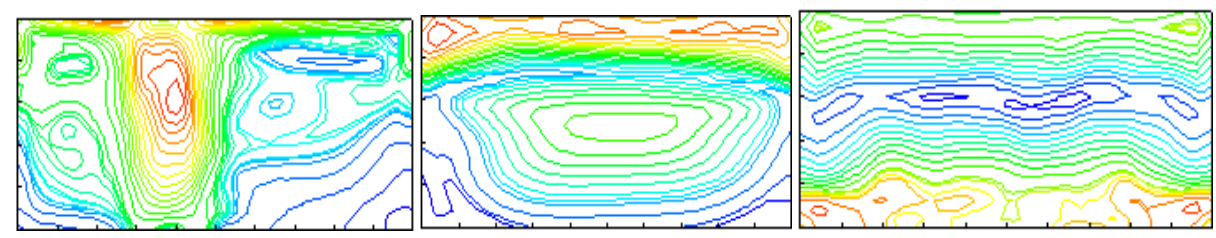

b) $\mathrm{S} 1(\mathrm{j}), U=0 \mathrm{~m} / \mathrm{s}$
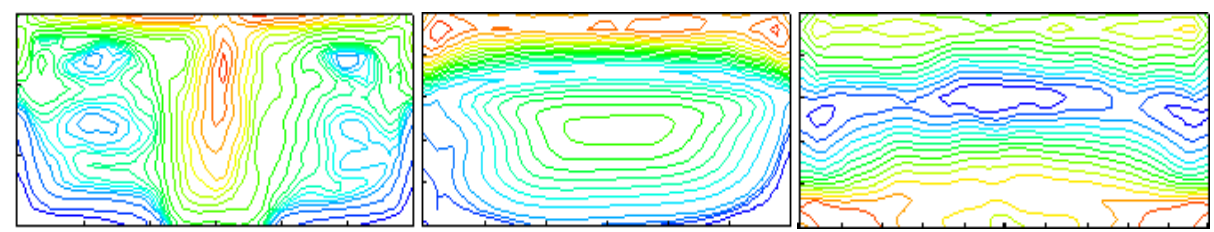

c) S0 (i), $U=0 \mathrm{~m} / \mathrm{s}$
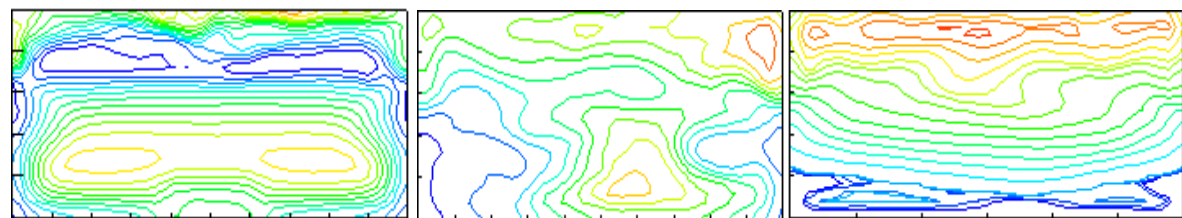

d) S0 (i), $U=0.23 \mathrm{~m} / \mathrm{s}$ 


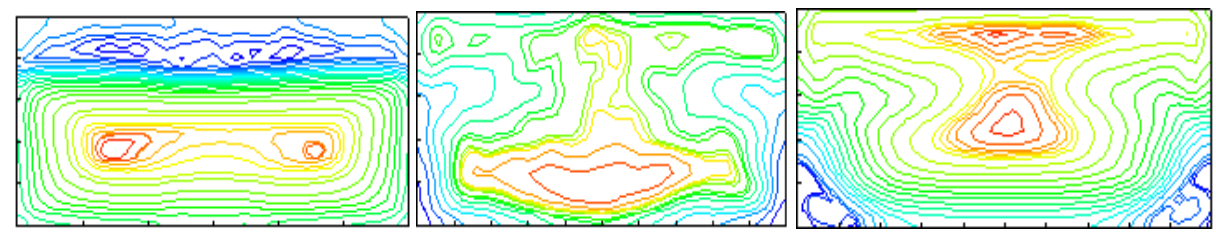

e) $\mathrm{S} 0(\mathrm{i}), U=0.46 \mathrm{~m} / \mathrm{s}$

Fig. 12. Vortex fields with different transversal position of the source, then the longitudinal velocity $(x=7.5,9$ and $14.5 \mathrm{~m})$.

Fig. 12 shows the structure of the flow at different sections of the downstream side of the canal. Figs. 12a, 12b and 12c show the effect of the variation of the transverse position of the source without the longitudinal air flow. The effect of the variation of the position of the source with a longitudinal air flow is shown in Figs. 12d and 12e.

In the absence of longitudinal air flow, there is the presence of the two recirculation zones on both sides of the plume $(x=7.5 \mathrm{~m})$. By moving the source of the wall, an asymmetry of the size of the vortices is observed. If the source is close to the wall, a recirculation zone is exhausted from the other side and forms a vortex below the ceiling to a height $(h / 2)$.

Advancing to the section $x=9 \mathrm{~m}$, the flow becomes similar and independent of the position of the source. There is a layer of smoke flowing below the ceiling with another recirculation zone that defines the entrained air. Mixed layer intermediary is not visible.

At the downstream section of the channel, $x=14.5 \mathrm{~m}$, a mixing occurs between the lower and upper layers, representing three horizontal layers, smoke layer, mixed layer and the layer of entrained air. Mixing the intermediate zone, where is product the destractification flow, turbulence is subject to the combined actions of the shear between the two flows and gravity forces acting on the smoke layer.

Applying a longitudinal air flow, Fig. 12d, a smoke layer below the ceiling is observed. At $z<0.2 \mathrm{~m}$, there are two symmetric vortices. Downstream, the flow is homogeneous. By increasing the longitudinal velocity until $U=0.46 \mathrm{~m} / \mathrm{s}$, Fig. 12e, there is the formation of a pair of counter-rotating vortices limits attached on both sides of the plume. in downstream, the flow is homogeneous up to the final section.

The plume is inclined under the effect of the dynamic pressure exerted by the longitudinal velocity. Its envelope undergoes a structural change characterized by an overpressure zone (upstream). Downstream after passing plume shear occurs at the reaction zone generating gravitational instabilities giving rise to the formation of three-dimensional structures. Finally, the inclination of the plume is characterized by its angle of deflection from the vertical. When a fire is subjected to a longitudinal velocity, the plume structure is modified both in the region close to the source as well as in the far field with little influence.

Vertical profiles of the velocity of the smoke propagation according to the longitudinal speed are shown in Fig. 13 at different slopes. In the absence of longitudinal air flow, the propagation is symmetrical relative to the ignition source for a zero slope. Smoke layer flows with a velocity equal to $0.2 \mathrm{~m} / \mathrm{s}$, the profiles allow inflection points that can inform us about the stratification of the flow. 
In the case where the channel is steep, the profiles are reversed between the upstream and downstream for two opposite slopes $\left(-2.5^{\circ}, 2.5^{\circ}\right)$. Also the values of the flow velocity are almost identical, $0.7 \mathrm{~m} / \mathrm{s}$, the profile is vertical up to the height $0.12 \mathrm{~m}$.

By applying a longitudinal air flow, $U=0.23 \mathrm{~m} / \mathrm{s}$, it is found that the velocity profile admits an inflection point at the upstream side of the canal. This indicates the existence of two layers flow, when the slope is zero. The training speed increases to $0.5 \mathrm{~m} / \mathrm{s}$ relative to the case where there is not a longitudinal air flow. At downstream channel, profile tends to be flattened, $(0.3 \mathrm{~m} / \mathrm{s})$, because the flow is controlled by the velocity of the longitudinal air flow.

If the channel is inclined at a slope of $2.5^{\circ}$, the velocity of the flow takes the form of continuous profile upstream of the channel; the whole quantity is transported to the downstream channel. Downstream, the speed of the smoke flow increases to $0.7 \mathrm{~m} / \mathrm{s}$. This behavior is inversely proportional to the upstream and downstream channel for slopes equal to $2.5^{\circ}$ and $-2.5^{\circ}$.

By increasing the velocity, $U=0.46 \mathrm{~m} / \mathrm{s}$, the profiles have the same characteristics as the air flow stream applied. They are continuous and almost flat upstream and downstream of the channel for all cases.
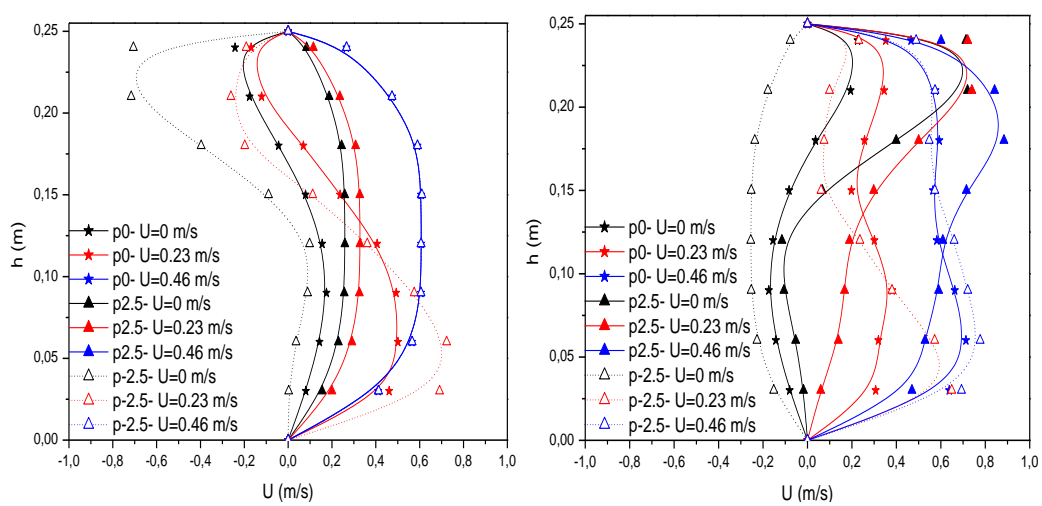

Fig. 13. Velocity profile $(x=5,10 \mathrm{~m})$.
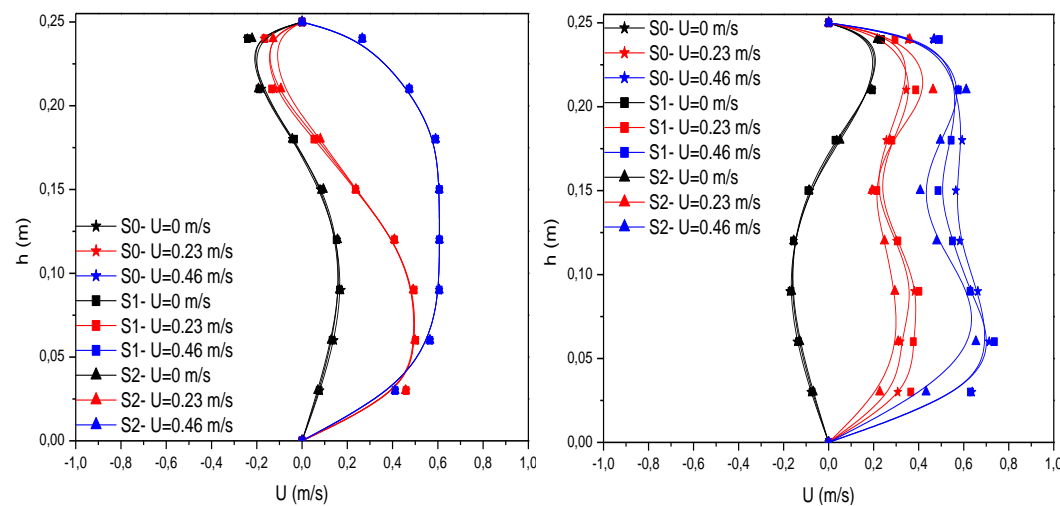

Fig. 14 . Velocity profile $(x=5,10 \mathrm{~m})$. 
Fig. 14 shows the vertical profiles of the flow velocity by setting the transverse position of the source. The smoke spread symmetrically in both directions at a speed of about 0.2 $\mathrm{m} / \mathrm{s}$. The speed of the smoke reaches $4 \mathrm{~m} / \mathrm{s}$ to scale with a power of $10 \mathrm{MW}$. There is, also, that as it progresses, the speed of the smoke layer decreases. A layer of fresh air remains below the layer of smoke; it is driven in the direction of the fire. By increasing the longitudinal velocity, the flow is accelerated until the velocity profile becomes flat.

In confined spaces such as tunnels, there is a phenomenon of accumulation of smoke to the ceiling (Figs. 15 and 16), governed by the dynamic and gravitational characteristic. The plume of smoke comes crashing down from the ceiling and spreads radially if the environment is at rest as shown in Figs. 12a and 16. The development of the flow in the ceiling is stopped by physical boundaries.

In addition, the tunnel walls generate a unidirectional flow of the smoke under the ceiling. When longitudinal air flow is applied to the plume, it causes the tilt and actually increases the amount of air entrained. Therefore, the speed of the flow from the plume increases. By increasing the intensity of the air stream, the plume behaves like a pseudoobstruction (Figs. 15 and 16).

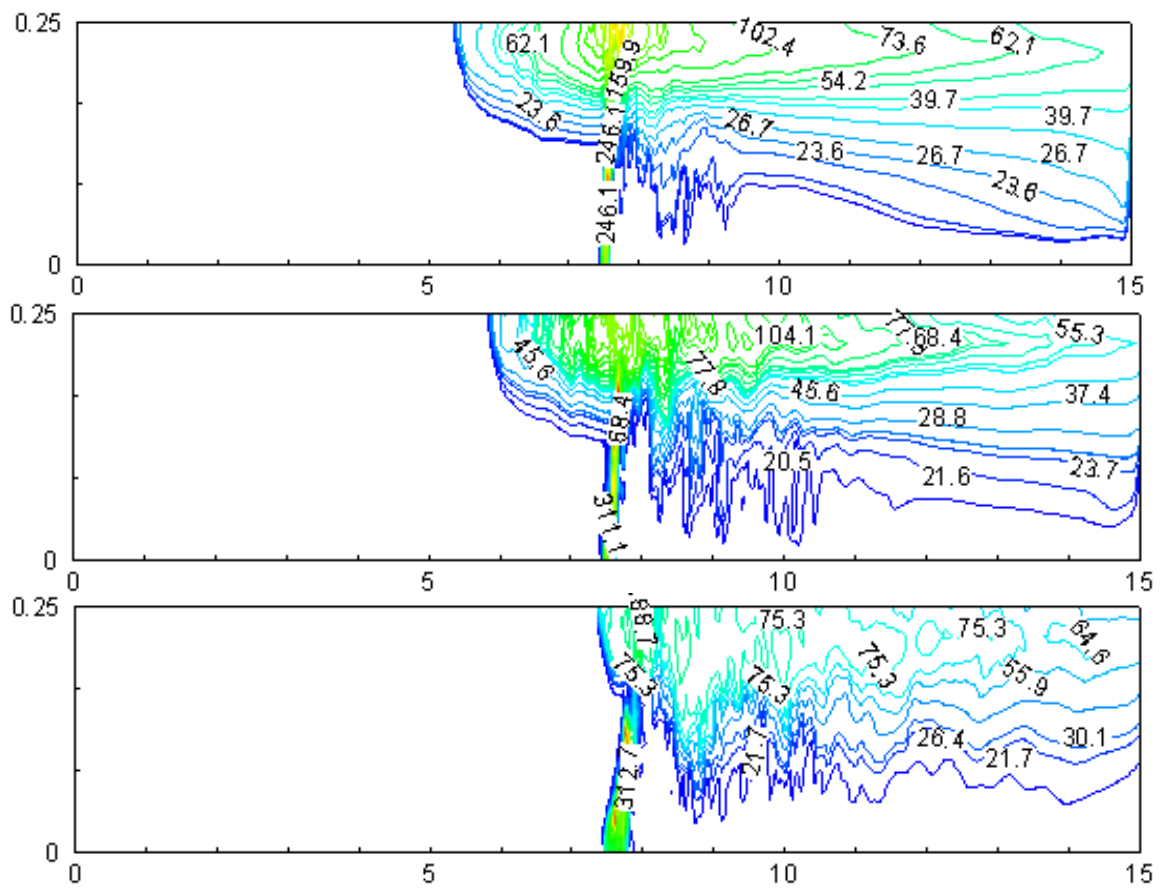

Fig. 15 . Temperature fields for a slope equal to $2.5^{\circ}$, a) $U=0 \mathrm{~m} / \mathrm{s}$; b) $U=0.23 \mathrm{~m} / \mathrm{s}$; c) $U=0.46 \mathrm{~m} / \mathrm{s}$.

Layer of smoke that forms under the ceiling temperature properties are relatively homogeneous as shown in Fig. 15a. This is due to turbulent mixing in the air recirculation zone of plume and the smoke layer. The recorded temperature values reach $350{ }^{\circ} \mathrm{C}$ at the plume, and $75{ }^{\circ} \mathrm{C}$ to $120{ }^{\circ} \mathrm{C}$ below the ceiling. 
The decrease in temperature is observed in the vertical direction. Depending on the length of the tunnel, the smoke layer Unidirectional then drops it approaches ground level. By applying a longitudinal air flow, stratified smoke layer will gradually dissolve when the longitudinal ventilation increases gradually. On the upstream side of the fire, a backlayering smoke is created. On the downstream side of the fire, the smoke stratification is governed by the heat losses to the turbulent mixing. $U=0.23 \mathrm{~m} / \mathrm{s}$ (Fig. 15b), the smoke layer below the ceiling is still visible. At the downstream channel, the values of the temperature are $30{ }^{\circ} \mathrm{C}$ to $50{ }^{\circ} \mathrm{C}$ in the intermediate layer. Layer smoke flows with temperatures of $50{ }^{\circ} \mathrm{C}$ to $120{ }^{\circ} \mathrm{C}$. $U=0.46 \mathrm{~m} / \mathrm{s}$ (Fig. 15c), the total amount of smoke is convected in downstream channel. The temperature distribution is uniform because the longitudinal velocity is the one that controls the flow.

In Fig. 16a, the smoke spread almost symmetrically (with load losses and drafts natural) under the effect of gravity (force temperature). The smoke is maintained in the upper part of the tunnel with values of temperature of $470{ }^{\circ} \mathrm{C}$. It degrades depending on the tunnel length symmetrically. There is, also, that as it progresses, the layer of smoke is cooled and its speed decreases. Continuing to move away, the smoke closer to the ground and may be returned to the plume by the airflow otherwise. The temperature field, in downstream of the source, presented the oscillations.

By applying a longitudinal velocity $U=0.23 \mathrm{~m} / \mathrm{s}$ (Fig. 16b), the temperature distribution in the tunnel is not symmetrical. Upstream of the source, there is a zone of interaction or flow is disrupted as shown in the figure. The temperature does not exceed $100{ }^{\circ} \mathrm{C}$. Downstream, the temperature is higher than in the first case and is distributed into the upper half of the tunnel. By increasing the speed up to $U=0.46 \mathrm{~m} / \mathrm{s}$ (Fig. 16c), the temperature is that of the ambient air upstream of the channel. Downstream, the temperature values are close, which means the flow homogenization. Temperature decreases compared to the case without ventilation. The back-layering is canceled and there is a slight turbulence upstream of the impact of smoke at the ceiling.

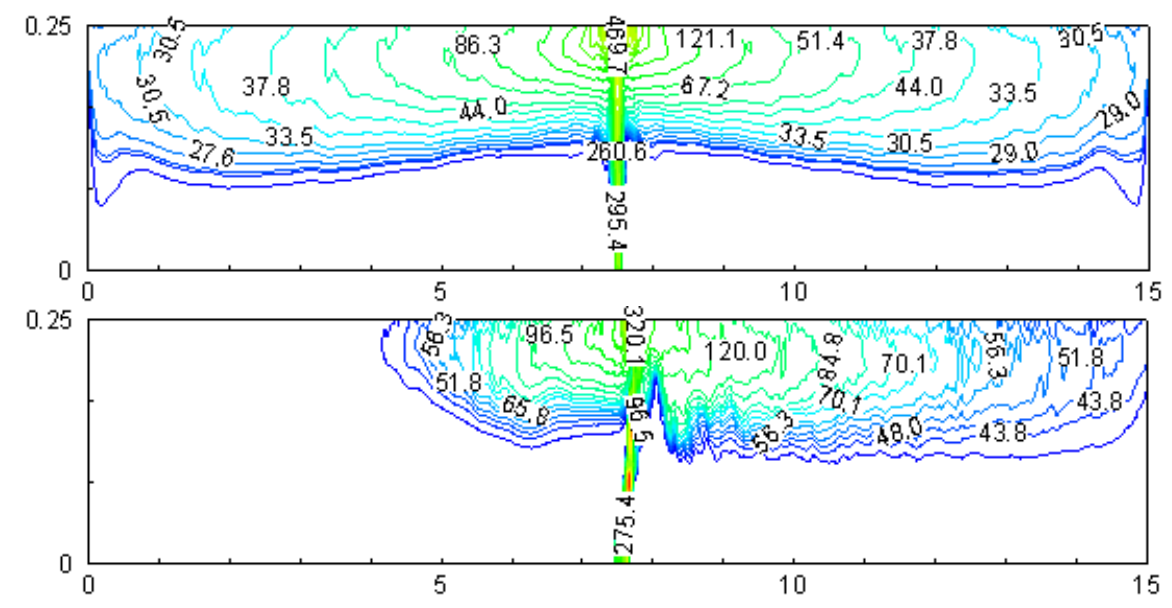




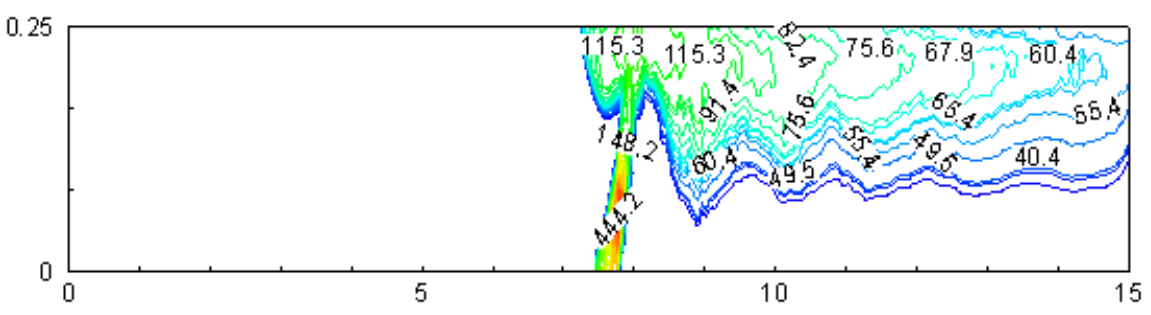

Fig. 16. Temperature fields, $y=0.25$, $S 0$ (i), a) $U=0 \mathrm{~m} / \mathrm{s} ; \mathrm{b}) \mathrm{U}=0.23 \mathrm{~m} / \mathrm{s} ; \mathrm{c}) \mathrm{U}=0.46 \mathrm{~m} / \mathrm{s}$

To quantify these results of stratification, using the Richardson number « $R \mathbf{i} »$ this is as follows:

$$
R \mathrm{i}=\frac{g \beta \Delta T L_{C}}{U^{2}}
$$

Note that this number is the ratio of the buoyancy (temperature gradient) and inertia (velocity) of the flow. In Figs. 17 and 18, the Richardson number is a function of the dimensionless length.

The longitudinal velocity is important with regard to the stratification and the smoke propagation. Note that the velocity profile influences the evolution of the Richardson number. Variations in the thickness of the mixing layer to depend mainly on the kinematics movement related to shear.

The Richardson number is around 10, when there is no longitudinal air flow, with a slight disturbance throughout the channel as shown in Figs. 17a and 18b. Based also on the profiles of the velocity, which admits an inflection point for the case study, we can conclude that the flow is stratified. Note also that the slope promotes the stratification of the flow as it shown in Fig. 17a.

Applying a longitudinal velocity of $0.23 \mathrm{~m} / \mathrm{s}$ (Figs. 17b and 18b), upstream, a peak is recorded the Richardson number which reflects the mixture of air and the longitudinal layer of smoke. A smoke stratification is likely to continue. Upstream, the flow undergoes oscillation until it reaches the source region with $R \mathrm{i} \approx 13$. On the other hand, due to the low speed, smoke stratification is not significantly disturbed. The Richardson number is below 10.

By increasing the velocity, $U=0.46 \mathrm{~m} / \mathrm{s}$, the entire quantity is transported to the downstream channel and the Richardson number is zero for a slope equal to $2.5^{\circ}$ in the upstream channel. Downstream, the profiles tend to flatten. The flow is controlled by the longitudinal velocity. Richardson number tends to values less than 10 and the flow is homogeneous view of the importance of inertia in all cases. 

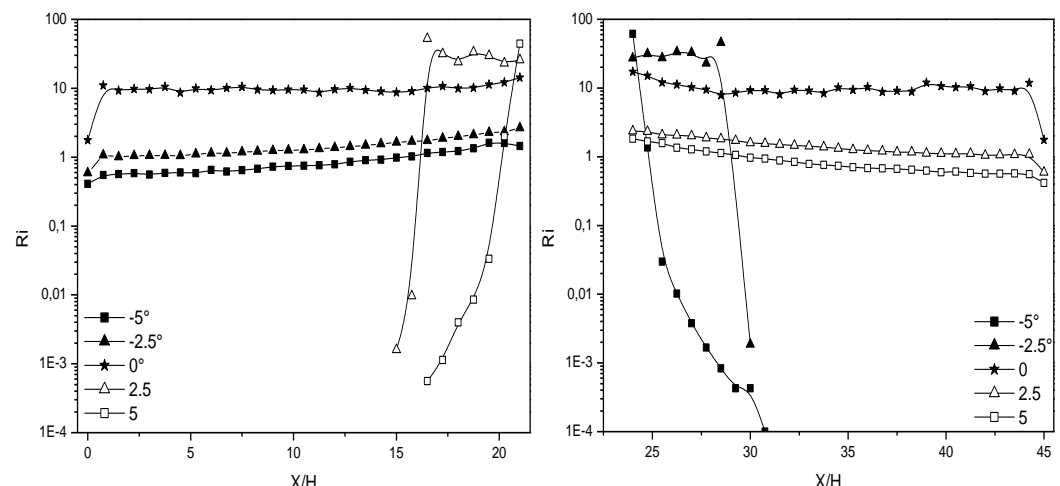

Fig.17a. Richardson number function the dimensionless length.
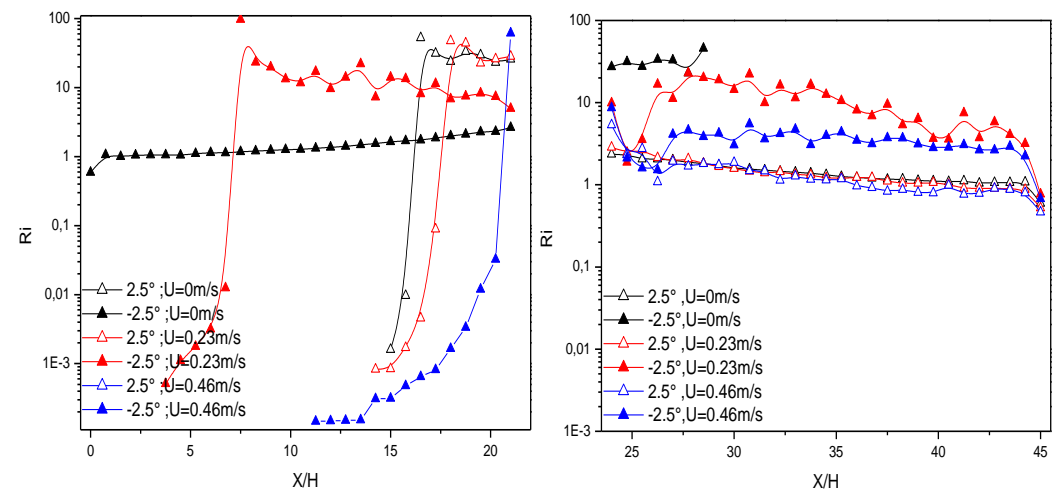

Fig.17b. Richardson number function the dimensionless length.
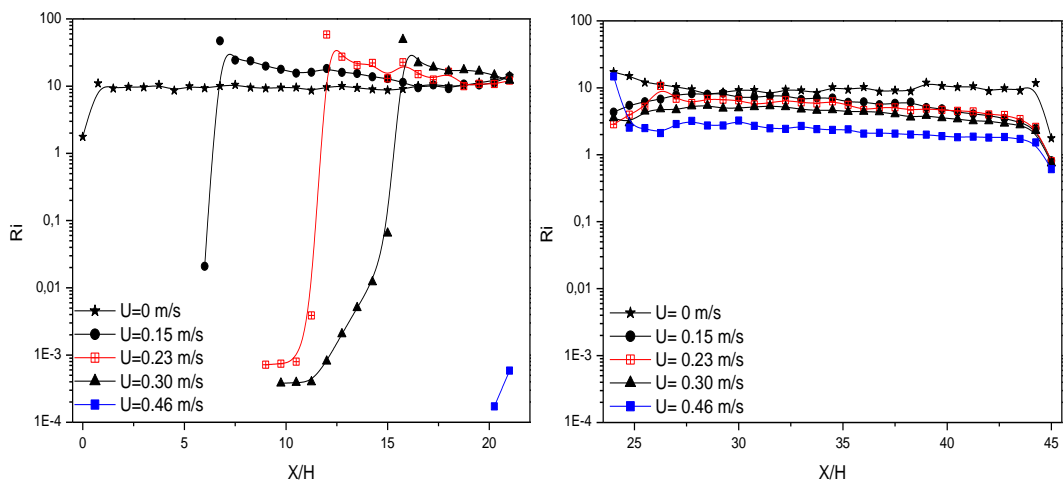

Fig.18. Richardson number function the dimensionless length.

\section{Conclusion}

The study of the structure and stratification of the flow is analyzed in this paper with the FDS code that showed a great performance. The slope is a slight influence on the structure 
of the phase unidirectional flow. The big change is made to the area of the source (plume). Fumes spread much more quickly to the direction for a high tunnel slope, in the absence of longitudinal air flow, the slope promotes flow stability.

In the presence or absence of air flow, the results showed that the transversal position of the source does not affect the structure of the flow, especially in the phase unidirectional. The flow is symmetrical in terms of thermal and dynamic compared to the source. The velocity of the longitudinal air is a great influence on the temperature distribution along the tunnel, the maximum temperature in the tunnel would be reduced, and the smoke stratification is dispatched when the longitudinal speed becomes greater. The results showed that the flow is stable when the Richardson number is around 10 and they could be useful for the design of control strategies smoke in tunnels.

\section{References}

1. J. P. Kunsch, Atmos. Environ. 33, 13 (1999). http://dx.doi.org/10.1016/S1352-2310(98)00118-6

2. J. P. Kunsch, Fire Safety J. 37 (1), 67 (2002). http://dx.doi.org/10.1016/S0379-7112(01)00020-0

3. K. Brahim, B. Mourad, E. C. Afif, and B. Ali, J. Appl. Fluid Mech. 6 (1) 49 (2013).

4. L. Junmei, L. Shanshan, L. Yanfeng, C. Chao, L. Xuan, and Y. Chenchen, Procedia Eng. 45, 690 (2012). http://dx.doi.org/10.1016/j.proeng.2012.08.224

5. R. Ballesteros-Tajadura, C. Santolaria-Morros, and E. Blanco-Marigorta, Tunnel. Undergr. Space Techn. 21, 21 (2006). http://dx.doi.org/10.1016/j.tust.2005.04.006

6. G. T. Atkinson and Y. Wu, Fire Safety J. 27 (4), 335 (1996). http://dx.doi.org/10.1016/S0379-7112(96)00061-6

7. S. R. Lee, H. S. Ryou, Build. Environ. 41, 719 (2006). http://dx.doi.org/10.1016/j.buildenv.2005.03.010

8. L. H. Hu, R. Huo, Y. Z. Li, H. B. Wang, and W. K. Chow, Tunnel. Undergr. Space Techn. 20, 223 (2005). http://dx.doi.org/10.1016/j.tust.2004.08.007

9. J. Ji, C. G. Fan, W. Zhong, X. B. Shen, and J. H. Sun, Intern. J. Heat Mass Trans. 55, 4817 (2012). http://dx.doi.org/10.1016/j.ijheatmasstransfer.2012.04.052

10. C. G. Fan, J. Ji, Z. H. Gao, J. Y. Han, and J. H. Sun, Intern. J. Heat Mass Trans. 56, 750 (2013). http://dx.doi.org/10.1016/j.ijheatmasstransfer.2012.09.047

11. E. Blanchard, P. Boulet, S. Desanghere, E. Cesmat, R. Meyrand, J. P. Garo, and J. P. Vantelon, Fire Safety J. 47, 18 (2012). http://dx.doi.org/10.1016/j.firesaf.2011.09.009

12. Y. ZhenLi, BoLei, HaukurIngason, Fire Safety J. 46, 204 (2011).

13. D. Yang, L. H. Hu, R. Huo, Y. Q. Jiang, S. Liu, and F. Tang, Appl. Therm. Eng. 30, 872 (2010). http://dx.doi.org/10.1016/j.applthermaleng.2009.12.019

14. F. Chen, Appl. Mech. Rev. 53, 207 (2000). http://dx.doi.org/10.1115/1.3097350

15. K. McGrattan, S. Hostikka, J. Floyd, H. Baum, R. Rehm, W. Mell, R. McDermott, Fire Dynamics Simulator (Version 5) Technical Reference Guide (NIST Special Publication 1018-5, June 23, 2010).

16. K. McGrattan, R. McDermott, S. Hostikka, J. Floyd, Fire Dynamics Simulator (Version 5) User's Guide (NIST Special Publication 1019-5, June 23, 2010).

17. Y. Wu and M. Z. A. Bakar, Fire Safety J. 35(4), 363 (2000). http://dx.doi.org/10.1016/S0379-7112(00)00031-X 